\title{
AN ANALYSIS OF SUPPLY CHAIN DECISIONS WITH ASYMMETRICAL RETAILERS: EFFECTS OF DISRUPTIONS AND STATIC SERVICE COST ON COORDINATION MECHANISM
}

\author{
Kebing Chen $^{1}$, Lei YAng ${ }^{2}$ And YAnXia LiU ${ }^{1}$
}

\begin{abstract}
The risk of demand or production cost disruption is one of the challenging problems in the supply chain management. This paper explores a generalized supply chain game model incorporating the possible disruptions. We find that a nonlinear Grove wholesale price scheme can fully coordinate such a supply chain even when both market demand and production cost are disrupted. The nonlinear Grove wholesale price scheme has three sides to coordinate the decision behavior of the players. One is that the mechanism can induce the retail pricing decided by the dominant retailer to be equal with that of the channel; the second is that the mechanism can induce fringe retailers to be not priced out of the market; the third is that the mechanism can ensure that the manufacturer uses minimum incentives to induce the dominant retailer to sell its product as well as providing the demandstimulating service. Disruptions from both demand side and production cost side may also affect the wholesale price, order quantity as well as retail price, and the share of the dominant retailer and the subsidy rate provided by the manufacturer are unity of opposites. We also find that it is optimal for the manufacturer to keep the original production plan when the joint-disruption amount is sufficiently small.
\end{abstract}

Keywords. Supply chain management, coordination, disruption management, game theory, robustness.

Mathematics Subject Classification. 90B60, 90 B50.

\footnotetext{
Received September 12, 2008. Accepted June 15, 2012.

1 College of Science, Nanjing University of Aeronautics and Astronautics, Nanjing, Jiangsu 210016, P.R. China. kbchen@nuaa.edu.cn

2 School of Economics and Commerce, South China University of Technology, Guangzhou, Guangdong 510006, P.R. China. yanglei2208@163.com
} 


\section{INTRODUCTION}

The risk of demand disruption is one of the challenging problems in operations management. It has been well recognized that the customers' demand is possibly affected by a number of factors such as the selling price, the demand-stimulating service, and others. Generally, a supply chain is coordinated when the players, acting rationally, make the decisions that are optimal for the whole supply chain. Some coordination mechanisms, such as revenue-sharing scheme and quantity discount scheme can be used to regulate the relationship among the supply chain's members. Very often, schemes are designed for the static environment such as a known market demand, or a distribution function in the stochastic environment. These schemes can be defined as static coordination mechanism. After the production plan have been agreed, the marketing environment can often be disrupted by unexpected haphazard events, which may result in the change of the demand or production cost to certain extent. For example, the outbreak of earthquake can cause a large sudden demand for tent; the epidemic of mad cow disease can affect a large degree of the demand for the beef consumption. There are other kinds of emergency that have made great tragedy on human beings such as the power blackouts in August 2003 in North America and tsunami natural disasters on Indian Ocean in 2005.

The probability of all these unexpected events' occurring is very small, however, the consequence is very serious. As reported in [26], disruptions from accidents in the chemical industry have led to huge economic losses and environmental damages, from the Bhopal and Exxon Valdez disasters, to the hundreds of lesser events that continue to occur on a yearly basis. A representative example is the March 2000 fire at the Philips microchip plant in Albuquerque, New Mexico. That plant supplied chips to Ericsson. However, the fire caused by lighting led to the disruption of supply to Ericsson. Since Ericsson was sourcing only from that plant, it could not avoid a production shutdown. As a result, Ericsson suffered $\$ 400$ million in lost sales. Additionally, Robert [35] reported that the labor strike at two General Motors parts plants in 1998 led to the shutdowns of 26 assembly plants and ultimately prevented the company from building over 500000 vehicles and led to a $\$ 809$ million quarterly loss. Therefore, the disruptions of environment would lead to huge loss for the whole system.

Additionally, companies such as Ericsson, Hershey, Apple, Wal-Mart, and a host of other major companies who rely on timely delivery of products and services to meet customer needs have incurred major losses due to supply chain disruptions. Moreover, small scale disruptions occur much more frequently, while the disruptions have made companies aware of the need for active disruption management.

In our paper, we consider coordination of a supply chain consisting of one manufacturer, one dominant retailer and multiple fringe retailers after possible disruptions. In practical operations within a supply chain, there often is a dominant retailer in the retail channel, which can be described as the well-known dominant retailer model (see [34,37]). For example, Wal-Mart's sale volume accounted 
for $39 \%$ of Tandy's in 2002. While, with its $\$ 285$ billion sales in 2004, Wal-Mart dwarfed any other competitors and became one of the largest companies in the world [31]. In such a retail channel, the dominant retailer often is a price leader and a main or largest distributor of its supplier. Other fringe retailers can be regarded as the price followers, and the market demand share of each fringe retailer is very small [44]. Additionally, we assume that only the dominant retailer can provide the advertising service to promote sales in such a retail channel. In general, the selling price has a well-understood impact on the customer's purchase decision, and the demand-stimulating service factor may be more complicated. We will investigate how the demand-stimulating service affects the decision of the dominant retailer and how to design the coordination mechanism by addition of the demand-stimulating service even if both the demand and the production cost are disrupted.

Differed from game model in the normal operation (i.e., without disruption), the sudden change of demand or production cost will cause certain deviation costs in the irregular operation (i.e., with disruption). That is, the deviation penalty is incorporated into the utility functions of each player or the channel decision-maker in the irregular operation, e.g., see $[7,45]$. For the multiple competing retailers, we regard that the fringe retailers are identical and the changed amount of the demand is common knowledge to all players in order to simplify the analysis. In our coordination mechanism, we assume that deviation costs are shared by both the manufacturer and dominant retailer. For the irregular operation, when the demand suddenly increases, the manufacturer should produce more products ordered by the retailers, which can result in extra deviation charge; additionally, when the demand decreases suddenly, the manufacturer may cost extra to handle the redundant production.

This paper mainly studies how to coordinate the supply chain with a dominant retailer when both the demand and the production cost are disrupted. To adopt optimal decisions to deal with disruptions from the channel's perspective would give distinctive advantages and coordination direction to decision-makers in the decentralized channel. For the case of competing retailers, most literatures assume that the retailers are symmetrical, and study the corresponding pricing decision and coordination mechanism. Few literatures consider the coordination mechanism for asymmetrical retailers. The main contribution of this paper lies in the following three sides.

(1) The coordination mechanism is designed for the supply chain with the asymmetrical competing retailers.

(2) The coordination mechanism induces the retail pricing of dominant retailer to be equal with that of the channel, simultaneously, ensures that fringe retailers are not priced out of the market. The mechanism ensures that the manufacturer uses minimum incentives to induce the dominant retailer to sell its product as well as providing the demand-stimulating service.

(3) The optimal wholesale price for the dominant retailer is decreasing with the disrupted demand, but increasing with the disrupted production cost. 
The wholesale price for each fringe retailer is independent of disrupted production cost. Additionally, the share of dominant retailer and the subsidy rate provided by the manufacturer are unity of opposites.

The remainder of this paper is organized as follows. Section 2 presents the related literature, and the basic model and centralized decision under the normal operation are investigated in Section 3. Section 4 considers coordination mechanism for normal operation with a Grove wholesale price scheme. Section 5 studies the corresponding scheme for the irregular operation. These analytical results are illustrated by numerical examples in Section 6. Finally, in Section 7, we summarize the results and point out possible directions for the future research.

\section{LiterATURE REVIEW}

This study is related to the intersection of coordination mechanism and disruption management in a supply chain compatible with multiple retailers and stimulating service. As we all know, the optimal supply chain profit is usually not achieved due to double marginalization in the decentralized decisions. Double marginalization usually results in the optimal order quantity for the buyer being lower than the optimal order quantity in a coordinated profit-maximizing supply chain. It occurs when the manufacturer marks up product to a wholesale price above manufacturing cost thereby reducing the potential retail margin.

Designing coordination scheme has been an important issue aimed at reconciling conflicts and achieves a better coordination among players. The excellent introduction and summaries on coordination management can be found in [5], however, it does not consider pricing decisions under the demand-stimulating service. In [30], it assumes that there is a duopoly facing no demand uncertainty and investigates a symmetric Nash Bargaining model, and it shows that Nash Bargaining can lead to higher supply chains profits than a vertically integrated chain. In [50], it develops a game model to synchronize different processing times through strategic placement of safety stocks at each player's premise, and finds that a revenue sharing contract can be used to coordinate the decentralized channel with the uncertain demand. In [19], it proposes charging the worse performing supplier a penalty and shows that such a mechanism is able to coordinate the assembly system with uncertain component yields and demand. In [11], it is assumed that a decentralized supply chain is consisting of a manufacturer and a retailer where the retailer simultaneously determines the retail price and order quantity, and it shows that the type of easy-to-implement agreement can achieve perfect supply chain coordination. Additionally, a buyback contract is an arrangement whereby the manufacturer agrees to provide the retailer credit for unsold product, see [13]. Similar literatures on coordination mechanisms can be also found in $[1,27,42]$, etc.

An important scheme presented in our paper is similar to the nonlinear wholesale price scheme studied in [6], which compares the wholesale price scheme with the revenue-sharing scheme, and finds that the two schemes are equivalent for 
the supply chain with a manufacturer and a retailer. In [4], it explores coordination issues in a supply chain with one wholesaler and multiple independent dispersed retailers and shows that the channel members will improve the channel and distribute the gains of coordination through bargaining. However, it has not provided a specific mechanism to coordinate such a supply chain. Further, a quantity discounts scheme is provided to coordinate the supply chain with multiple independent retailers (see [9]), and a nonlinear wholesale pricing scheme is also provided to coordinate the supply chain with symmetrical competing retailers, (see [3]). For the multiple competing retailers, however, there are few literatures on the design of coordination mechanism when considering the demand disruption, not to mention the asymmetrical competing retailers. Differing from current literatures, we will investigate how to design a coordination mechanism to coordinate the supply chain with multiple asymmetrical competing retailers when the demand is disrupted, and study how the service cost and the demand disruption affect the coordination mechanism.

In this paper, we develop a single-period game model of supply chain with a dominant retailer to investigate the optimal decisions of the players, where the demand-stimulating service is incorporated into the model. Recent studies have investigated carriers or provider issues and their effects on consumer services and operating strategies. The seller can use some advertising programs to convince customers to purchase their products [21]. In [29], it considers the coordination issue in a decentralized supply chain composed of one vendor and one buyer, in which the vendor can offer a single product to the buyer who is faced with service level constraint. For the co op advertising service [10] investigates the combined effects of the co op advertising mechanism, the return policy and the channel coordination, and shows that the cooperative decision policy is always found to be superior to the non-cooperative decision policy in terms of profit improvement. In [36], it is assumed that the demand is influenced by both price and advertisement, and it considers vertical coop advertising along with pricing decisions in a supply chain consisting of one manufacturer and one retailer. Comparison results reflect the significant effect the shape of demand-price function may have on optimal values of decision variables and supply chain members' profit. In [47], it investigates the channel coordination by seeking optimal co op advertising strategies and equilibrium pricing in a two-member distribution channel, and identifies the feasible solutions to a bargaining problem where the channel members can determine how to divide the extra profits. Other literatures about co op advertisement can be also referred, e.g., in $[2,14,20,23-25,39,48]$.

At the same time, our paper mainly investigates the decisions of dominant retailer. The term of 'dominant' implies that a channel player can have the power of controlling or influencing another member's decisions. Current literatures considering the dominant-retailer models often assume that the retailer has stronger bargaining power than that of the manufacturer, and is a Stackelberg leader in the channel, for example, see $[16,28,40]$, etc. Differing from the current assumption, we assume that the manufacturer is Stackelberg leader in the channel in our paper, 
while among the multiple competing retailers there is a main or largest retailer of the supplier, this retailer has higher market power. Similar assumption can be found in $[12,33,43]$. In [33], it is assumed that the supply chain is consisting of the supplier and a dominant retailer, and shows that such a supply chain can be coordinated to the benefit of the manufacturer through either quantity discounts or a menu of two-part tariffs. It also sheds light on the role of 'street money' in channel coordination and shows that such a practice can arise from a manufacturer's effort to mete out minimum incentives to engage the dominant retailer in channel coordination. In [12], it considers the coordination mechanism of the supply chain consisting of a dominant retailer. However, they do not consider how the service cost and the disrupted production cost affect the coordination mechanism.

Additionally, the disruption management in a supply chain is another aspect closely related to our work. Supply chain disruption management has increasingly attracted attentions. The concept of disruption management was first introduced in [15]. One main difference between the coordination under disruptions and the coordination under normal environment is that the sudden changes of demands and costs will cause certain deviation costs that do not exist before. For the literatures on the disruption management, the coordination problem of supply chain consisting of one manufacturer and one retailer after demand disruption is considered, see [29]. Usually, the disruption can result in extra cost. In order to simplify the models, the deviation cost is sometimes assumed to be borne by the manufacturer or shared by both players [46] extends the model into the supply chain with two competing retailers who have the options of investment on sales promotion, where the demand is the function of the promotion investment decisions. In [49], it studies the impacts of supply disruption on the supply chain system by using simulation approach in which two different distribution function of random variable were used to express the supply disruption. The disruption management can also be found in two-stage production and inventory system, see [45]. Additionally [38] presents a broad range of models for designing supply chains that are resilient to disruptions. Similar literatures can also be found in $[17,22]$. However, the above literatures do not consider the disruption management for the asymmetrical competing retailers. Differing from the current literatures, therefore, this paper will mainly investigate how to coordinate the supply chain with the asymmetrical competing retailers when both cost and demand are disrupted.

\section{THE BASIC MODEL}

We model a distribution channel consisting of one manufacturer selling its product through one dominant retailer and $N$ fringe symmetrical retailers, where $N \geqslant 2$. The unit production cost of the manufacturer is denoted as $c$. We assume that the dominant retailer is a price leader and a largest distributor of the manufacturer and only the dominant retailer can provide the demand-stimulating service, which goes beyond what the fringe retailers can do. For example, the dominant retailer can carry on some propagating advertisement to promote the 
product of manufacturer. Let $s_{f}$ be the demand-stimulating service given in the demand, and the corresponding cost of providing the demand-stimulating service is denoted as $f$. In the selling process, we also assume that the dominant retailer has the decision right of pricing and providing the demand-stimulating service or not. Once the retail price is settled down by the dominant retailer, all fringe retailers regard it as the market retail price, which is generally consistent with the marketing operation described earlier where some small retailers use the pricing book of a large retailer, and similar studies can be found in literatures such as [44].

When the dominant retailer provides the demand-stimulating service, we assume that the total market demand can be written as following.

$$
q_{T}=a-b p+s_{f}
$$

In the above demand function, $a$ is the market scale, and $a>0 . b$ is denoted as the price sensitive coefficient, and $p$ is the retail price, which is decided by the dominant retailer. Similar assumption on the demand can be found in $[33,42]$. Here, we assume $a+s_{f}>b c$ to ensure that the order quantity is nonnegative. The total demand $\left(q_{T}\right)$ for the manufacturer's product depends on the price charged by the dominant retailer. The dominant retailer should pay service investment cost, denoted as $f$, which will yield $s_{f}$-unit demand. From equation (3.1), this demand function implies that the demand is decreasing with retail price but increasing with a fixed value by the service investment. Similar to [33], both $s_{f}$ and $f$ are assumed to be static parameters. Additionally, we assume that the total market demand in the final market without providing the service is denoted as $q_{T, n}$.

In the retail channel, we assume that the share of the market demand for the dominant retailer is denoted as $\gamma$, and then the market demand for the dominant retailer can be denoted as $q_{d}=\gamma q_{T}$, and that for each fringe symmetrical retailer can be denoted as $q_{r}=(1-\gamma) q_{T} / N$. That is, the demand-stimulating service provided by the dominant retailer can also be beneficial to the each fringe retailer. In order to simplify the analysis, we assume that the reservation utilities of the retailers are all zero. That is, each retailer including the dominant retailer will like to participate as long as they can gain a nonnegative profit.

First, we first study the centralized decision in which a central planner makes all decisions to maximize the expected channel profit to provide a benchmark for the normal operation. The profit of centralized supply chain should be $\pi_{T}=$ $q_{T}(p-c)-f$. Solving the first-order condition of $\pi_{T}$ for $p$, we can derive the optimal retail price of channel, which can be denoted as $p^{*}=\left(a+s_{f}+b c\right) /(2 b)$. Obviously, the retail price increases with respect to the market scale and the unit production cost. Inserting the optimal retail price into (3.1) can yield the optimal production quantity i.e., $q_{T}^{*}=\left(a+s_{f}-b c\right) / 2$ for the manufacturer, and then we can obtain the corresponding optimal profit of whole supply chain, which can be denoted as $\pi_{T}^{*}=\left(a+s_{f}-b c\right)^{2} /(4 b)-f$.

The retail price or the order quantity of centralized channel gives the evaluation criterion for the coordination mechanism when the supply chain is under the decentralized operation. In order to ensure the dominant retailer to provide the 
corresponding demand-stimulating service, we should make the following assumptions.

\section{Assumption 3.1.}

(i) The fixed cost of providing demand-stimulating service should satisfy the condition as $f \leqslant f_{0}$, where $f_{0}=\gamma s_{f}\left[s_{f}+2(a-b c)\right] /(4 b)$;

(ii) The dominant retailer's market share should satisfy the inequality as $1>\gamma \geqslant$ $1 /(N+1)$.

Part (i) ensures that the inequality, i.e., $\gamma q_{T}^{*}\left(p^{*}-c\right)-f \geqslant \max \gamma(a-b p)(p-c)$, can be satisfied. It means that the profit of dominant retailer is so large that the dominant retailer would like to provide the demand-stimulating service, and the profit-driven manufacturer can also have motivation to induce such service even when the supply chain is in the decentralized operation. For Part (ii), we can give the following explanation. Since we assume that the dominant retailer is the largest distributor of the manufacturer, and the share of dominant retailer should be larger than the average market share of each retailer. That is, the value of $\gamma$ indicates the absolute market power for the dominant retailer, who behaves as a monopolist in setting its price and in deciding whether to provide the demand-stimulating service investment (see [33]). Such a supply chain can be coordinated by different schemes. In the following, we will investigate how to realize the channel coordination by the nonlinear Grove wholesale price scheme for the normal operation as well as the irregular operation when the demand is disrupted.

\section{CoORdinAtion MECHANism FOR NORMAL OPERATiON}

The decentralized supply chain will be coordinated if the dominant retailer exactly sets the retail price equaling to the value obtained in centralized system and provides the desired service. In order to achieve the supply chain coordination, the manufacturer can also use a pricing mechanism to implement quantity discount. Similar to $[3,18]$, the coordination mechanism aims to align each retailer's decisions with those of the supply-chain-wide. Based on this idea, a simple channel-coordinating Grove wholesale price can be designed.

Let the unit wholesale price charged by the manufacturer be $w\left(q, s_{f}\right)$ when the order quantity of a retailer is $q$ and the cost of service provision of the dominant retailer is $f$. Under the nonlinear Grove wholesale price scheme, we denote the profits of dominant retailer, each symmetrical fringe retailer and manufacturer as $\pi_{d}, \pi_{r}$ and $\pi_{m}$, respectively. The profit of dominant retailer can be denoted as following

$$
\pi_{d}=\left(p-w\left(q_{d}, s_{f}\right)\right) q_{d}-f .
$$

If the nonlinear Grove wholesale price mechanism provided by the manufacturer can ensure that the retailers' decisions are aligned with the decisions of the centralized decision-maker, and then the supply chain is coordinated. In the nonlinear Grove wholesale price, we assume that the dominant retailer can obtain a certain 
percentage of the channel' profit, and the dominant retailer's share is denoted as $\lambda_{1}$, where $0<\lambda_{1}<1$. To encourage each retailer to order more products and induce the dominant retailer to provide the demand-stimulating service for their customers, one of the feasible solutions for the manufacturer is to offer subsidy policies to the dominant retailer. And then, we let $\lambda_{2}$ be the subsidy rate provided by the manufacturer in a nonlinear Grove wholesale price scheme to the dominant retailer, where $0<\lambda_{2}<1$. Both the parameters $\lambda_{1}$ and $\lambda_{2}$ are independent of retail price or order quantity.

This alignment can be realized by specifying the wholesale pricing function $w\left(q, s_{f} \mid \lambda_{1}, \lambda_{2}\right)$ if the dominant retailer's profit is transformed into the following.

$$
\pi_{d}=\lambda_{1}\left(a-b p+s_{f}\right)(p-c)-\left(1-\lambda_{2}\right) f
$$

The parameters $\lambda_{1}$ and $\lambda_{2}$ decide that the nonlinear Grove wholesale price mechanism can arbitrarily allocate the total profit between the manufacturer and the retailers, and the split of channel's profit depends on the players' relative bargaining power. Usually, the manufacturer, acting as the Stackbelberg leader, can have stronger bargaining ability than that of the followers. For example, in the monopolist market, the manufacturer can choose retailers to sell its product, and the manufacturer will have stronger power in the bargaining problem. So the manufacturer should take as much profit from all retailers as possible. Of course, the mechanism provided by the manufacturer should ensure that each retailer would have interest to participate, that is, each retailer should obtain the reservation utility in the scheme, which gives the direction of the coordination mechanism. In the following, we will study how to design the mechanism to coordinate such a supply chain.

First, we should determine the traded wholesale price. From equations (4.1) and (4.2), it is obvious that the following wholesale price scheme can ensure that the dominant retailer choose the optimal order quantity of the centralized supply chain.

$$
w\left(q, \gamma \mid \lambda_{1}, \lambda_{2}\right)=\left(1-\frac{\lambda_{1}}{\gamma}\right)\left(\frac{a+s_{f}}{b}-\frac{q}{b \gamma}\right)-\left(\frac{\lambda_{2} f}{q}-\frac{\lambda_{1} c}{\gamma}\right) .
$$

For the wholesale price presented in equation (4.3), the first term means that when the dominant retailer's order quantity is $q$ and the investing level is $f$ in service, the dominant retailer should pay a variable fee per unit. The second term means that the dominant retailer should receive a lump sum compensation of $\lambda_{2} f / q$, however, he should also bear part of the manufacturer's production cost, $\lambda_{1} c / \gamma$. The fringe retailers will simply pay the variable fee of $w\left(q, \gamma \mid \lambda_{1}, \lambda_{2}\right)$ without the second term. Additionally, the manufacturer should take as much profit away from the competitive fringe retailers as possible. It is important for the manufacturer to choose the proper $\left(\lambda_{1}, \lambda_{2}\right)$ to achieve it. That is, our scheme is consisting of three important parameters, i.e. $\left(w, \lambda_{1}, \lambda_{2}\right)$, to decide the mechanism's feasibility.

We evaluate the supply chain scheme by two criteria. First, the scheme should guarantee supply chain coordination, i.e., it can maximize the profit of the whole 
supply chain. Otherwise, it will likely perform sub-optimally. Second, the scheme should guarantee that the profit of each player should be no less than the reservation utilities, i.e., the profit of each player under the supply chain scheme should be no less than the reservation utility. The second criterion is very critical. If the scheme can guarantee the supply chain coordination but cannot assure the second condition, then this scheme will be infeasible because achieving coordination may result in one of the parties to be worse off. The following proposition makes it clear that such a scheme is feasible and unique.

Theorem 4.1. Under the nonlinear Grove wholesale price scheme $\left(w, \lambda_{1}, \lambda_{2}\right)$, we have:

(i) the supply chain with a dominant retailer can be coordinated by the wholesale price $w\left(q, \gamma \mid \lambda_{1}, \lambda_{2}\right)=\left(1-\frac{\lambda_{1}}{\gamma}\right)\left(\frac{a+s_{f}}{b}-\frac{q}{b \gamma}\right)-\left(\frac{\lambda_{2} f}{q}-\frac{\lambda_{1} c}{\gamma}\right)$;

(ii) the dominant retailer and each fringe retailer's profits are all increasing with $\lambda_{1}$ linearly;

(iii) the optimal decisions $\left(\lambda_{1}^{*}, \lambda_{2}^{*}\right)$ for the manufacturer should be denoted as

$$
\begin{gathered}
\lambda_{1}^{*}=\gamma \frac{(N \gamma+\gamma-1)\left(a-b c+s_{f}\right)}{(2 N \gamma+\gamma-1)\left(a+s_{f}\right)+b c(1-\gamma)}, \\
\lambda_{2}^{*}= \begin{cases}0, & 0 \leqslant f \leqslant \lambda_{1}^{*} \frac{s_{f}\left[s_{f}+2(a-b c)\right]}{4 b} \\
1-\lambda_{1}^{*} \frac{s_{f}\left[s_{f}+2(a-b c)\right]}{4 b f}, & \lambda_{1}^{*} \frac{s_{f}\left[s_{f}+2(a-b c)\right]}{4 b} \leqslant f \leqslant f_{0} ;\end{cases}
\end{gathered}
$$

(iv) $\quad \frac{\mathrm{d} \lambda_{1}^{*}}{\mathrm{~d} \gamma}>0$ and $\frac{\mathrm{d} \lambda_{2}^{*}}{\mathrm{~d} \gamma} \leqslant 0$.

Part (i) of Theorem 4.1 shows that such a nonlinear Grove wholesale price can achieve the channel coordination for the supply chain with a dominant retailer. That is, the dominant retailer will order the quantity of centralized channel. Part (ii) implies that the dominant retailer's bargaining power also affects the benefit of fringe retailers, that's, all fringe retailers will be benefited from the increase of the bargaining ability of dominant retailer. Part (iii) indicates that the higher the service cost, the higher the subsidy rate provided by the manufacturer in the current scheme will be. Part (iv) implies that the higher the market share of the dominant retailer, the higher the dominant retailer's share of channel profit will be, however, the smaller subsidy will be provided by the manufacturer. That is, the dominant retailer's share in the current coordination mechanism has the reverse function with that of the subsidy rate, and there exist a restrictive interaction between these two factors in determining the profit of dominant retailer.

So far, we have designed a nonlinear Grove wholesale price mechanism that can gain an overall optimal performance of the entire supply chain under the normal operation. To extend the range of possible disruptions, we will investigate the corresponding coordination mechanism for the irregular operation in the next section, and analyze how disruptions from demand and production cost and service cost affect coordination mechanism. 


\section{CoORdination MECHANism FOR IRREgular OPERATION}

In the actual operation, there are many occasional events that will result in the change of demand or production cost suddenly. The coordinated scheme designed under the static case may become invalid, and then the supply chain should be re-coordinated when the demand is disrupted. With a certain unpredicted demand change, the players will make decisions to minimize their total cost or maximize their total profits when incorporating deviation costs. In general, the existing literature assumed that the deviation costs are incurred to the manufacturer, for example [8,32], etc. Differing from the current literatures, we assume that the dominant retailer shares deviation costs with the manufacturer. Since the retailer is directly facing with the changed demand and production cost, it will result in charge an extra fee by additional order quantity or return surplus items, and both the manufacturer and each retailer will face the disruptions from demand side and production cost side. Therefore, it is reasonable to assume that both the manufacturer and the dominant retailer should bear the deviation costs. We use the notation with a tilde $(\sim)$ to denote the case when the demand and the cost are disrupted.

\subsection{Centralized Supply Chain With Demand AND COST DisRuptions}

In a centralized supply chain system, the manufacturer and the retailers are vertically integrated in a supply chain, and they seek to maximize the total system profit of the supply chain after the disruptions are occurred. Here, we assume that the market scale $a$ is changed into $\tilde{a}=a+\Delta a>0$ and the cost of manufacturer $c$ is changed into $\tilde{c}=c+\Delta c>0 . \Delta a>0(\Delta a<0)$ indicates the increased (decreased) market demand. $\Delta a$ is independent of the demand-stimulating service. $\Delta c>0(\Delta c<0)$ indicates the increased (decreased) production cost. Then, the total demand function can be denoted as $\tilde{q}_{T}=\tilde{a}-b \tilde{p}+s_{f}$.

The changed demand will result in a production deviation, which is denoted as $\Delta q=\tilde{q}_{T}-q_{T}^{*}$. When the production deviation is larger than zero, i.e., $\Delta q>0$, more products should be produced to meet the unplanned increased quantity, which will result in an extra cost such as the extra machine, labor overtime. Otherwise, there is some leftover inventory which will result in an extra cost such as holding cost for the unused products. In either case, the demand disruption will cause disruptions to the original production plan and deviation penalties associated with the difference between the original planned quantity and the actual quantity beyond the planned resources may be required, and should be included when making the new price and production decisions. Similar to [29], we assume a unit penalty cost $c_{u} \geqslant 0$ for a unit increased quantity and a unit penalty cost $c_{s} \geqslant 0$ for a unit decreased quantity. Based on the above assumption, the total profit of the centralized supply chain should be

$$
\tilde{\pi}_{T}=\tilde{q}_{T}(\tilde{p}-c-\Delta c)-f-c_{u}\left(\tilde{q}_{T}-q_{T}^{*}\right)^{+}-c_{s}\left(q_{T}^{*}-\tilde{q}_{T}\right)^{+},
$$


where $(x)^{+}=\max \{x, 0\}$. The first two terms represents the total profit of the supply chain when both the demand and the cost are disrupted; the third (fourth) term presents the total penalty cost incurred by the increased (decreased) product. Generally speaking, some restraint conditions should be given for unit penalty cost of the decreased production, see [32]. In order to simplify the analysis, we give the following assumption.

Assumption 5.1. The unit penalty cost for decreased production is less than the unit production profit, i.e., $0<c_{s}<c+\Delta c+s / b$.

Assumption 5.1 means that the unit cost of the decreased production should be less than a certain value, that's to say, if the unit penalty cost for decreased production is too high, the manufacturer would have less production plan since it is not profitable to produce. Similar to the deduction of reference [32], we derive the following:

Theorem 5.2. The optimal decisions of the centralized supply chain with the demand and production cost disruptions can be denoted as the following three cases:

(i) when $\Delta a-b \Delta c \geqslant b c_{u}$, the optimal retail price and order quantity are $\tilde{p}_{\text {case } 1}^{*}=p^{*}+\frac{c_{u}}{2}+\frac{\Delta a+b \Delta c}{2 b}$ and $\tilde{q}_{T, \text { case } 1}^{*}=q_{T}^{*}-\frac{b c_{u}}{2}+\frac{\Delta a-b \Delta c}{2}$, respectively;

(ii) when $-b c_{s}<\Delta a-b \Delta c<b c_{u}$, the optimal retail price and order quantity are $\tilde{p}_{\text {case } 2}^{*}=p^{*}+\frac{\Delta a}{b}$ and $\tilde{q}_{T \text {, case } 2}^{*}=q_{T}^{*}$, respectively;

(iii) when $\Delta a-b \Delta c \leqslant-b c_{s}$, the optimal retail price and order quantity are $\tilde{p}_{\text {case } 3}^{*}=p^{*}-\frac{c_{s}}{2}+\frac{\Delta a+b \Delta c}{2 b}$ and $\tilde{q}_{T, \text { case } 3}^{*}=q_{T}^{*}+\frac{b c_{s}}{2}+\frac{\Delta a-b \Delta c}{2}$, respectively.

Assumption 5.1 ensure that the decision of retail price is nonnegative. From Proposition 5.7, we find that when the joint-disruption amount (i.e., $\Delta a-b \Delta c$ ) is very small, i.e., $-b c_{s}<\Delta a-b \Delta c<b c_{u}$, the optimal production quantity of manufacturer will be unchanged. That is, the deviation penalties can result in the robustness of production plan. The corresponding scale of the changed demand can be regarded as a robust scale. Theorem 5.1 gives the evaluation criterion of decentralized channel coordination when both the demand and the production cost are disrupted, simultaneously.

\subsection{Supply Chain CoOrdination With Demand AND COSt DisRuptions}

From Section 4, we know that a nonlinear Grove wholesale price scheme can be used to coordinate such a supply chain in the normal environment. Here, we investigate whether this scheme can coordinate such a supply chain consisting of one dominant retailer after the market scale is disrupted. For the given wholesale price, the profit of dominant retailer can be denoted as following function when both the demand and the production cost are disrupted.

$$
\tilde{\pi}_{d}=\left(\tilde{p}-\tilde{w}\left(\tilde{q}_{d}, \tilde{s}_{f}\right)\right) \tilde{q}_{d}-f .
$$

Here, we will consider the case that the manufacturer shares the disruption risk with the dominant retailer, that is, the dominant retailer will bear some part of 
the deviation costs. Similar to the coordination mechanism designed in the normal operation, the profit of the dominant retailer under the irregular operation can be denoted as follows

$\tilde{\pi}_{d}=\tilde{\lambda}_{1}\left[\left(\tilde{a}-b \tilde{p}+\tilde{s}_{f}\right)(\tilde{p}-\tilde{c})-c_{u}\left(\tilde{a}-b \tilde{p}+\tilde{s}_{f}-q_{T}^{*}\right)^{+}-c_{s}\left(q_{T}^{*}-\tilde{a}+b \tilde{p}-\tilde{s}_{f}\right)^{+}\right]-\left(1-\tilde{\lambda}_{2}\right) f$

And we can get the following unit wholesale price

$$
\begin{aligned}
\tilde{w}\left(\tilde{q} \mid \tilde{\lambda}_{1}, \tilde{\lambda}_{2}\right)= & \left(1-\frac{\tilde{\lambda}_{1}}{\gamma}\right)\left(\frac{\tilde{a}+s_{f}}{b}-\frac{\tilde{q}}{b \gamma}\right) \\
& -\left[\frac{\tilde{\lambda}_{2} f}{\tilde{q}}-\frac{\tilde{\lambda}_{1} \tilde{c}}{\gamma}-\frac{\tilde{\lambda}_{1} c_{u}}{\gamma}\left(1-\frac{q_{d}^{*}}{\tilde{q}}\right)^{+}-\frac{\tilde{\lambda}_{1} c_{s}}{\gamma}\left(\frac{q_{d}^{*}}{\tilde{q}}-1\right)^{+}\right] .
\end{aligned}
$$

Similar to equation (4.3), the first part of equation (5.4) means that when the dominant retailer's order quantity is $\tilde{q}$ and the investing level is $f$ in service, the dominant retailer should pay a variable fee per unit to the manufacturer. The second part contains four terms: the first term means a lump sum compensation for the dominant retailer, the second term is part production cost borne by the dominant retailer, the third term and the fourth term are the unit deviation costs shared by the dominant retailer. That is, in order to receive lump sum compensation, the dominant retailer should bear part of production cost and deviation cost.

The fringe retailers don't receive a lump sum compensation cost and simply pay a variable fee per unit $\tilde{w}\left(\tilde{q}, \tilde{s}_{f} \mid \tilde{\lambda}\right)$ to the manufacturer without the second part, because they do not provide demand-stimulating service. As the design of mechanism, the manufacturer should take as much profit away from the fringe retailers as possible. Similar to Assumption 3.1, we make the following assumption.

Assumption 5.3. The disrupted amounts and the cost of the demand-stimulating service should satisfy the condition as $f \leqslant \tilde{f}_{0}(\Delta a, \Delta c)$, where $\tilde{f}_{0}(\Delta a, \Delta c)=$ $\tilde{s}_{f} \gamma\left[\tilde{s}_{f}+2(\tilde{a}-b \tilde{c})\right] /(4 b)$.

Assumption 5.3 implies that the following condition can be satisfied.

$$
\max _{\tilde{p}} \gamma \tilde{\pi}_{T}\left(\tilde{p} \mid \tilde{s}_{f}\right)-(1-\gamma) f \geqslant \max _{\tilde{p}} \gamma \tilde{\pi}_{T}\left(\tilde{p} \mid \tilde{s}_{f}=0\right)-(1-\gamma) f
$$

Theorem 5.1 gives optimal decisions of centralized supply chain with the three cases of demand disruption. For these disruption cases, the following Theorems 5.4-5.8 present the corresponding coordination mechanisms for the decentralized supply chain, respectively.

Case 1. $\Delta a-b \Delta c \geqslant b c_{u}$

When the joint-disruption amount satisfies the condition of $\Delta a-b \Delta c \geqslant b c_{u}$, we have 
Theorem 5.4. The manufacturer can coordinate the supply chain with a dominant retailer by offering a nonlinear Grove wholesale price scheme as $\left(\tilde{w}_{\text {case } 1}^{*}, \tilde{\lambda}_{1, \text { case } 1}^{*}, \tilde{\lambda}_{2, \text { case } 1}^{*}\right)$, where

$$
\begin{aligned}
& \tilde{w}_{\text {case } 1}^{*}=\left(1-\frac{\tilde{\lambda}_{1, \text { case } 1}^{*}}{\gamma}\right)\left(\frac{a+\Delta a+\tilde{s}_{f}}{b}-\frac{\tilde{q}}{b \gamma}\right)-\left[\frac{\tilde{\lambda}_{2, \text { case } 1}^{*} f}{\tilde{q}}\right. \\
& \left.-\frac{\tilde{\lambda}_{1, \mathrm{case} 1}^{*}(c+\Delta c)}{\gamma}-\frac{\tilde{\lambda}_{1, \mathrm{case} 1}^{*} c_{u}}{\gamma}\left(1-\frac{q_{d}^{*}}{\tilde{q}}\right)^{+}\right], \\
& \tilde{\lambda}_{1, \text { case } 1}^{*}=\gamma \frac{(N \gamma+\gamma-1)\left(a+\Delta a+\tilde{s}_{f}-b c_{u}-b(c+\Delta c)\right)}{(2 N \gamma+\gamma-1)\left(a+\Delta a+\tilde{s}_{f}\right)+(1-\gamma)\left(b c_{u}+b(c+\Delta c)\right)},
\end{aligned}
$$

and

$$
\tilde{\lambda}_{2, \text { case } 1}^{*}= \begin{cases}0 & 0 \leqslant f \leqslant \tilde{\lambda}_{1, \text { case } 1}^{*} \frac{\tilde{s}_{f}\left[\tilde{s}_{f}+2(\tilde{a}-b \tilde{c})\right]}{4 b} \\ 1-\tilde{\lambda}_{1, \text { case } 1}^{*} \frac{\tilde{s}_{f}\left[\tilde{s}_{f}+2(\tilde{a}-b \tilde{c})\right]}{4 b f} & \tilde{\lambda}_{1, \text { case } 1}^{*} \frac{\tilde{s}_{f}\left[\tilde{s}_{f}+2(\tilde{a}-b \tilde{c})\right]}{4 b}<f \leqslant \tilde{f}_{0} .\end{cases}
$$

From Theorem 5.4, we can find that the nonlinear Grove wholesale price scheme has three sides to coordinate the decision behavior of the players. One is that the mechanism should induce the retail pricing of the retailer to be equal to that of the channel, which can be realized by $\tilde{w}^{*}$; the second is that the mechanism should induce the fringe retailers to be not priced out of the market, which can be realized by $\tilde{\lambda}_{1}^{*}$; the third is that the mechanism should induce manufacturer uses minimum incentives to induce the dominant retailer to sell its product as well as provide the corresponding demand-stimulating service, which can be realized by $\tilde{\lambda}_{2}^{*}$. The optimal subsidy rate is also influenced by the service cost. Especially, the manufacturer will provide more subsidy rate to counterbalance higher service cost from sales promotion, however, if the service cost is sufficiently low, the manufacturer may not provide any subsidy to the retailer's service. Additionally, from the traded wholesale price, the dominant retailer should bear part of production cost as well as part of the deviation cost for increased quantity.

In the following, we will analyze the characteristics of the parameters $\tilde{\lambda}_{1}^{*}$ and $\tilde{\lambda}_{2}^{*}$, which are important factors in determining the dominant retailer's share of the whole supply chain with the demand disruption. Comparing decisions under the normal environment with those under irregular environment, we can derive the following corollary.

Proposition 5.5. When $\Delta a-b \Delta c \geqslant b c_{u}$, we have:

(i) $\frac{\partial \tilde{\lambda}_{1, \text { case } 1}^{*}}{\partial \Delta a}>0, \frac{\partial \tilde{\lambda}_{2, \text { case } 1}^{*}}{\partial \Delta a}<0, \frac{\partial \tilde{\lambda}_{1, \text { case } 1}^{*}}{\partial \Delta c}<0$ and $\frac{\partial \tilde{\lambda}_{2, \text { case } 1}^{*}}{\partial \Delta c} \geqslant 0$;

(ii) $\tilde{\lambda}_{1, \text { case } 1}^{*}<\lambda_{1}^{*}$ for $c_{u} \leqslant \Delta a<\left(a+\tilde{s}_{f}\right)\left(c_{u}+\Delta c\right) / c ; \tilde{\lambda}_{1, \text { case } 1}^{*}=\lambda_{1}^{*}$ for $\Delta a=$ $\left(a+\tilde{s}_{f}\right)\left(c_{u}+\Delta c\right) / c ;$ and $\tilde{\lambda}_{1, \text { case } 1}^{*}>\lambda_{1}^{*}$ for $\Delta a>\left(a+\tilde{s}_{f}\right)\left(c_{u}+\Delta c\right) / c$. 
Proposition 5.5 mainly describes the trend of the coordination strategy for the supply chain with the demand disruption. From Part (i), we can find that when joint-disruption amount increases greatly $\left(\Delta a-b \Delta c \geqslant b c_{u}\right)$, the share of the dominant retailer $\left(\tilde{\lambda}_{1}^{*}\right)$ is increasing with $\Delta a$, and decreasing with $\Delta c$. However, the subsidy rate $\left(\tilde{\lambda}_{2}^{*}\right)$ is decreasing with $\Delta a$, and increasing with $\Delta c$. It implies that when the demand is increasing greatly, the dominant retailer can potentially obtain more revenue, which can result in the less subsidy rate. Additionally, when the production cost increases, the channel's profit will naturally decrease, and the manufacturer can decrease the share of retailer, however, he should increase subsidy rate to ensure that the dominant retailer has interest to cooperation. In general, the share of the dominant retailer $\left(\tilde{\lambda}_{1}^{*}\right)$ and the subsidy rate $\left(\tilde{\lambda}_{2}^{*}\right)$ are unity of opposites. Part (ii) implies that the share $\left(\tilde{\lambda}_{1}^{*}\right)$ under the irregular operation has close relationship with that in the normal operation, which depends on the amount of changed demand. Specially, only when the changed amount of demand is larger than a threshold $\left(\left(a+\tilde{s}_{f}\right)\left(c_{u}+\Delta c\right) / c\right)$, the share of dominant retailer obtained in the irregular operation can be higher than that obtained in the normal operation.

Case 2. $-b c_{s}<\Delta a-b \Delta c<b c_{u}$

When the joint-disruption amount satisfies the condition of $-b c_{s}<\Delta a-b \Delta c<$ $b c_{u}$, we have

Theorem 5.6. The manufacturer can coordinate the supply chain with a dominant retailer by offering a nonlinear Grove wholesale price scheme $\left(\tilde{w}_{\text {case } 2}^{*}, \tilde{\lambda}_{1, \text { case } 2}^{*}, \tilde{\lambda}_{2, \text { case } 2}^{*}\right)$, where

$$
\begin{gathered}
\tilde{w}_{\text {case } 2}^{*}=\left(1-\frac{\tilde{\lambda}_{1, \text { case } 2}^{*}}{\gamma}\right)\left(\frac{\tilde{a}+\tilde{s}_{f}}{b}-\frac{\tilde{q}}{b \gamma}\right)-\left(\frac{\tilde{\lambda}_{2, \mathrm{case} 2}^{*} f}{\tilde{q}}-\frac{\tilde{\lambda}_{1, \mathrm{case} 2}^{*} \tilde{c}}{\gamma}\right), \\
\tilde{\lambda}_{1, \text { case } 2}^{*}=\gamma \frac{(N \gamma+\gamma-1)\left(a+s_{f}-b c\right)}{(2 N \gamma+\gamma-1)\left(a+s_{f}\right)+2 \Delta a N \gamma+(1-\gamma) b c},
\end{gathered}
$$

and

$$
\tilde{\lambda}_{2, \text { case } 2}^{*}= \begin{cases}0 & 0 \leqslant f \leqslant \tilde{\lambda}_{1, \text { case } 2}^{*} \frac{\tilde{s}_{f}\left[\tilde{s}_{f}+2(\tilde{a}-b \tilde{c})\right]}{4 b} \\ 1-\tilde{\lambda}_{1, \text { case } 2}^{*} \frac{\tilde{s}_{f}\left[\tilde{s}_{f}+2(\tilde{a}-b \tilde{c})\right]}{4 b f} & \tilde{\lambda}_{1, \text { case } 2}^{*} \frac{\tilde{s}_{f}\left[\tilde{s}_{f}+2(\tilde{a}-b \tilde{c})\right]}{4 b}<f \leqslant \tilde{f}_{0} .\end{cases}
$$

Differing from case 1, Theorem 5.6 implies that the traded wholesale price remains same form with that under the normal operation, when the joint-disruption amount is very small. The three parameters in this case also have similar functions and managerial insights with the case of $\Delta a-b \Delta c \geqslant b c_{u}$. The traded wholesale price implies that the dominant retailer should just bear part of production cost. From 
Theorem 5.6, we derive the following:

Proposition 5.7. When $-b c_{s}<\Delta a-b \Delta c<b c_{u}$, we have

(i) $\frac{\partial \tilde{\lambda}_{1, \text { case } 2}^{*}}{\partial \Delta c}=0, \frac{\partial \tilde{\lambda}_{2, \text { case } 2}^{*}}{\partial \Delta c} \geqslant 0, \frac{\partial \tilde{\lambda}_{1, \text { case } 2}^{*}}{\partial \Delta a}<0 ;$ and when $a>\left(\frac{2 N \gamma}{1-\gamma}+1\right) b c+$ $\left(\frac{N \gamma}{1-\gamma}-1\right) \tilde{s}_{f}, \frac{\partial \tilde{\lambda}_{2, \text { case 2 }}^{*}}{\partial \Delta a}>0$

(ii) $\tilde{\lambda}_{1, \text { case } 2}^{*}<\lambda_{1}^{*}$ for $0<\Delta a<c_{u} ; \tilde{\lambda}_{1, \text { case } 2}^{*}=\lambda_{1}^{*}$ for $\Delta a=0 ;$ and $\tilde{\lambda}_{1, \text { case } 2}^{*}>\lambda_{1}^{*}$ for $-c_{s}<\Delta a<0$.

Differing from case 1 , we find that the share of the dominant retailer $\left(\tilde{\lambda}_{1}^{*}\right)$ is decreasing in the robust scale. With the decreasing of the dominant retailer's share in this case, it can not result in the more subsidy rate $\left(\tilde{\lambda}_{2}^{*}\right)$ provided by the manufacturer, except that the demand scale satisfies the condition as $a>(2 N \gamma /(1-\gamma)+1) b c+(N \gamma /(1-\gamma)-1) s$. It implies that the coordination mechanism takes advantage of the favorable increased demand to generate as much profit as possible for the manufacturer.

Case 3. $\Delta a-b \Delta c \leqslant-b c_{s}$

When the joint-disruption amount satisfies the condition of $\Delta a-b \Delta c \leqslant-b c_{s}$, we have

Theorem 5.8. The manufacturer can coordinate the supply chain with a dominant retailer by offering a nonlinear Grove wholesale price scheme $\left(\tilde{w}_{\text {case } 3}^{*}, \tilde{\lambda}_{1, \text { case } 3}^{*}, \tilde{\lambda}_{2, \text { case } 3}^{*}\right)$, where

$$
\begin{aligned}
\tilde{w}_{\text {case3 }}^{*}= & \left(1-\frac{\tilde{\lambda}_{1, \text { case } 3}^{*}}{\gamma}\right)\left(\frac{a+\Delta a+\tilde{s}_{f}}{b}-\frac{\tilde{q}}{b \gamma}\right) \\
& -\left[\frac{\tilde{\lambda}_{2, \text { case3 } f}^{*}}{\tilde{q}}-\frac{\tilde{\lambda}_{1, \mathrm{case} 3}^{*}(c+\Delta c)}{\gamma}-\frac{\tilde{\lambda}_{1, \mathrm{case} 3}^{*} c_{s}}{\gamma}\left(\frac{q_{d}^{*}}{\tilde{q}}-1\right)\right], \\
\tilde{\lambda}_{1, \text { case } 3}^{*}= & \gamma \frac{(N \gamma+\gamma-1)\left(a+\Delta a+\tilde{s}_{f}-b(c+\Delta c)+b c_{s}\right)}{(2 N \gamma+\gamma-1)\left(a+\Delta a+\tilde{s}_{f}\right)+(1-\gamma)\left(b(c+\Delta c)-b c_{s}\right)},
\end{aligned}
$$

and

$$
\tilde{\lambda}_{2, \text { case } 3}^{*}= \begin{cases}0 & 0 \leqslant f \leqslant \tilde{\lambda}_{1, \operatorname{case} 3}^{*} \frac{\tilde{s}_{f}\left[\tilde{s}_{f}+2(\tilde{a}-b \tilde{c})\right]}{4 b} \\ 1-\tilde{\lambda}_{1, \operatorname{case} 3}^{*} \frac{\tilde{s}_{f}\left[\tilde{s}_{f}+2(\tilde{a}-b \tilde{c})\right]}{4 b f} & \tilde{\lambda}_{1, \operatorname{case} 3}^{*} \frac{\tilde{s}_{f}\left[\tilde{s}_{f}+2(\tilde{a}-b \tilde{c})\right]}{4 b}<f \leqslant \tilde{f}_{0} .\end{cases}
$$

From the traded wholesale price of this case, we can find that the dominant retailer will bear part of production cost as well as part of the deviation cost for the decreased quantity. From Theorem 5.8, we can derive the following 
Proposition 5.9. when $\Delta a-b \Delta c \leqslant-b c_{s}$, we have

(i) when $c_{s}=c+\Delta c, \frac{\partial \tilde{\lambda}_{1, \text { case } 3}^{*}}{\partial \Delta a}=0$ and $\frac{\partial \tilde{\lambda}_{2, \text { case } 3}^{*}}{\partial \Delta a}<0$; when $\forall c_{s} \in(0, c+\Delta c) \cup$ $\left(c+\Delta c, c+\Delta c+s_{f} / b\right), \frac{\partial \tilde{\lambda}_{1, \text { case } 3}^{*}}{\partial \Delta a}>0, \frac{\partial \tilde{\lambda}_{2, \text { case } 3}^{*}}{\partial \Delta a}<0 ; \frac{\partial \tilde{\lambda}_{1, \text { case } 3}^{*}}{\partial \Delta c}<0, \frac{\partial \tilde{\lambda}_{2, \text { case } 3}^{*}}{\partial \Delta c} \geqslant 0 ;$ (ii) $\tilde{\lambda}_{1, \text { case } 3}^{*}<\lambda_{1}^{*}$ for $-a<\Delta a<-\left(a+\tilde{s}_{f}\right)\left(c_{s}-\Delta c\right) / c ; \tilde{\lambda}_{1 \text {, case } 3}^{*}=\lambda_{1}^{*}$ for $\Delta a=$ $-\left(a+\tilde{s}_{f}\right)\left(c_{s}-\Delta c\right) / c ; \tilde{\lambda}_{1, \text { case } 3}^{*}>\lambda_{1}^{*}$ for $-b c_{s} \geqslant \Delta a>-\left(a+\tilde{s}_{f}\right)\left(c_{s}-\Delta c\right) / c$.

Differing from the previous two cases, we find that when the unit penalty cost for a unit decreased quantity satisfies $c_{s}=c+\Delta c$, the share of dominant retailer $\left(\tilde{\lambda}_{1}^{*}\right)$ is independent with the changed demand, see Part (i) of Proposition 5.9. However, with the increase of demand in this case, it will result in the less subsidy rate provided by the manufacturer for this scenario. On the other hand, when then unit penalty cost satisfies the condition as $c_{s} \neq c+\Delta c$, we find that the share $\left(\tilde{\lambda}_{1}^{*}\right)$ is increasing with the changed demand amount $\Delta a$. Similar to case 1 , we can also find that when the demand is increasing in case 3 , the dominant retailer can potentially obtain more revenue, which can result in the less subsidy rate provided by the manufacturer.

From Theorems 5.4-5.8, we can derive the following result.

Proposition 5.10. For each case, we always have $\tilde{\lambda}_{1, \text { case } i}^{*}<\gamma$, for $i=1,2,3$.

Proposition 5.10 implies that the dominant retailer will bear part of the manufacturer's production cost and the deviation cost for the sake of receiving lump sum compensation in each case of joint-disruption amount. From Theorems 5.4-5.8, in short, we can obtain the following two aspects managerial insights for each disruption case. First, the nonlinear Grove wholesales price scheme provided by the manufacturer can ensure that the fringe retailers are not priced out of the market. Second, the manufacturer uses minimum incentives to induce the dominant retailer to sell its product as well as provide the demand-stimulating service. Additionally, we can show that if the joint-disruption amount increases greatly $\left(\Delta a-b \Delta c \geqslant b c_{u}\right)$, the dominant retailer will obtain more profit than that in the normal environment; if the joint-disruption amount decreases greatly $\left(\Delta a-b \Delta c \leqslant-b c_{s}\right)$, the dominant retailer will obtain less profit than that in the normal environment; otherwise, the profit of the dominant retailer is unchanged, see the case of joint-disruption amount satisfying the condition as $-b c_{s}<\Delta a-b \Delta c<b c_{u}$.

\section{Numerical EXAMPLES}

In this section, we present some examples to analyze the effects of the demand disruption on the coordination mechanism to draw more managerial insights from the theoretical results above. We assume the originally estimated market scale $a=10$, the unit production cost of the manufacturer $c=1.5$, the demandstimulating service yields $s_{f}=2$, the price sensitive coefficient $b=1.5$, both deviation penalty $\operatorname{costs} c_{u}=1$ and $c_{s}=1$, respectively. The number of competing 


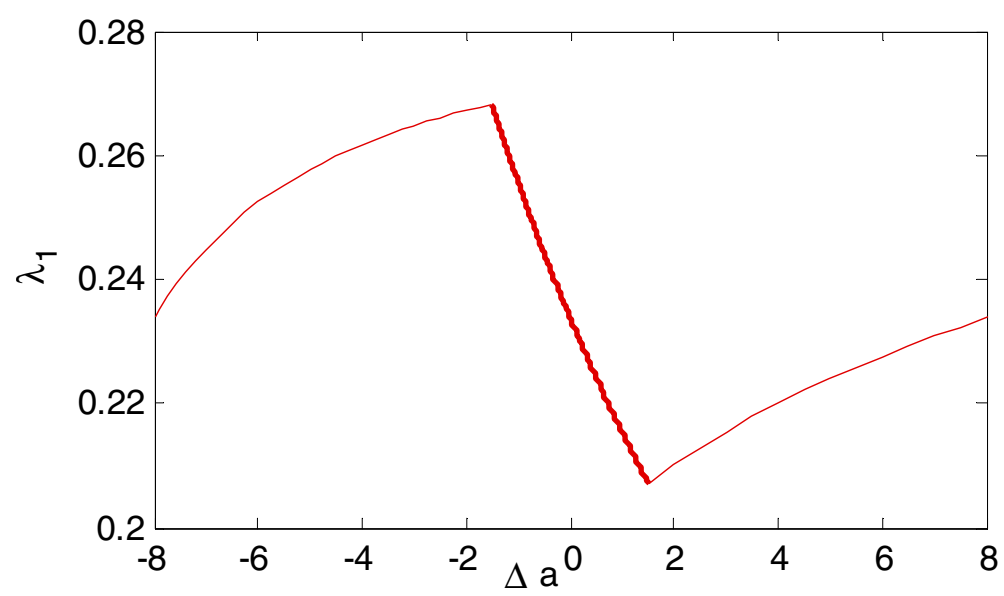

FiguRE 1. Optimal share of the dominant retailer versus demand disruption with $\Delta c=0$.

fringe retailer is denoted as $N=10$, and the share of the market demand for the dominant retailer is $\gamma=0.6$, and the cost of providing the demand-stimulating service, $f$, increases from 1 to 5 by a step 1, and the changed amount of demand, $\Delta a$, increases from -8 to 8 , and the changed amount of production cost, $\Delta c$, increases from -1 to 6 . we are interested in the effects of various disruption demands and demand-stimulating service cost on the channel's decisions and the coordinated nonlinear Grove wholesale price scheme.

First, we investigate the effect different disruption amount of demand on the retailers' optimal share of channel's profit when the unit production cost of the manufacturer is unchanged, which is depicted in Figure 1.

From Figure 1, we know that the larger the changed demand amount, the higher the optimal total order quantity in both cases 1 and 3 will be. However, when the disrupted amount of demand is small, i.e., case 2, the optimal total order quantity remains a fixed constant in this disrupted scale, which is equal with that obtained in the normal operation, that is, when the disrupted amount of demand is small, the original production plan of the manufacturer should not be changed. Figure 1 presents the relationship between the optimal share of dominant retailer and disrupted demand amount. In both cases 1 and 3, we can find that the shares $\left(\lambda_{1}^{*}\right)$ are increasing with the disrupted demand amount $(\Delta a)$. However, the entirely reverse relationship can be found for $\lambda_{1}^{*}$ and $\Delta a$ in the robust scale of case 2 . That is, the manufacturer takes some special measures to induce the fringe retailers to be not priced out of the market.

Second, we will investigate how the demand disruption effects on the optimal subsidy rate provided by the manufacturer. Table 1 presents the disrupt scale of demand under different demand-stimulating service costs $(f)$, and Figure 2 
TABLE 1. Optimal subsidy rate.

\begin{tabular}{|c|c|c|c|c|c|}
\hline & \multicolumn{5}{|c|}{ The scale of $\Delta a$ for $w^{*}, \lambda_{2}^{*}$ with $\Delta c=0$} \\
\hline$f$ & "none" & $1-\tilde{\lambda}_{1, \text { case } 3}^{*} \tilde{f}_{0} / \gamma$ & $1-\tilde{\lambda}_{1, \text { case } 2}^{*} \tilde{f}_{0} / \gamma$ & $1-\tilde{\lambda}_{1, \text { case } 1}^{*} \tilde{f}_{0} / \gamma$ & 0 \\
\hline 1 & {$[-8,-6.25]$} & {$[-6.25,-3.08]$} & - & - & {$[-3.08,8]$} \\
\hline 2 & {$[-8,-3.75]$} & {$[-3.75,-1.5]$} & {$[-1.5,1.5]$} & {$[1.5,4.71]$} & {$[4.71,8]$} \\
\hline 3 & {$[-8,-1.25]$} & - & {$[-1.25,1.5]$} & {$[1.5,8]$} & - \\
\hline 4 & {$[-8,1.25]$} & - & {$[1.25,1.5]$} & {$[1.5,8]$} & - \\
\hline 5 & {$[-8,3.75]$} & - & - & {$[3.75,8]$} & - \\
\hline
\end{tabular}

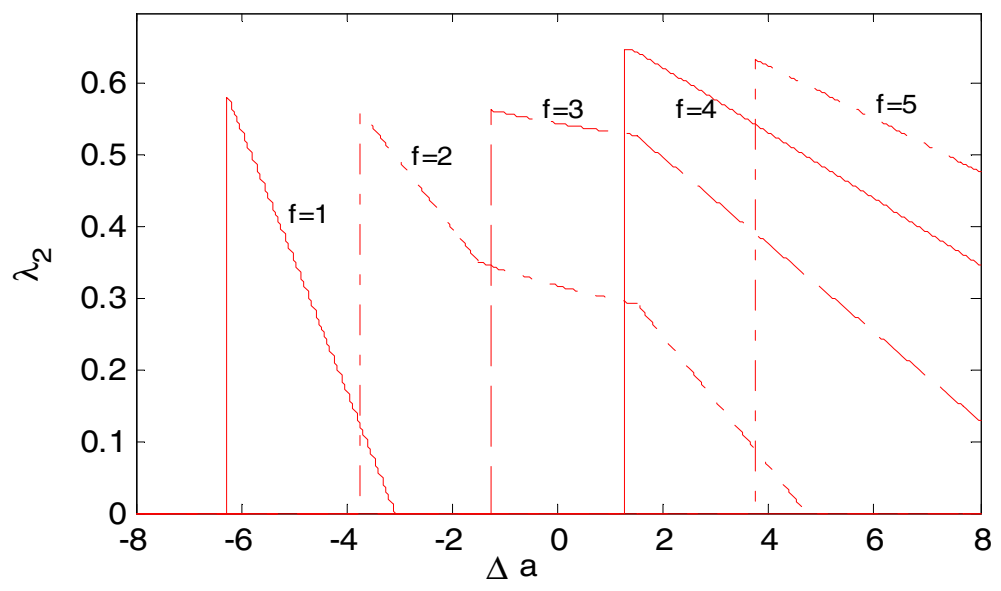

Figure 2. Optimal subsidy rate versus demand disruption with $\Delta c=0$.

illustrates how both the demand disruption and demand-stimulating service cost affect the optimal subsidy rate.

Remark 6.1. "None" denotes that the cost $f$, for the given scope of $\Delta a$ goes beyond its coordination range of the channel with providing the demand-stimulating service.

From Table 1, we can find that with the increase of the demand-stimulating service cost, the feasible region of the disruption amount $\Delta a$ becomes smaller. Intuitively, the channel will not have ability to provide expensive service cost if the demand is excessively low.

From Figure 2, we find that when the market demand decreases greatly or the cost of providing the demand-stimulating service is great, the manufacturer should increase subsidy rate to ensure that the dominant retailer has interest to cooperation and provision of retail services. That is, the mechanism should induce manufacturer uses minimum incentives to induce the dominant retailer to sell its product as well as providing the corresponding demand-stimulating service. When 


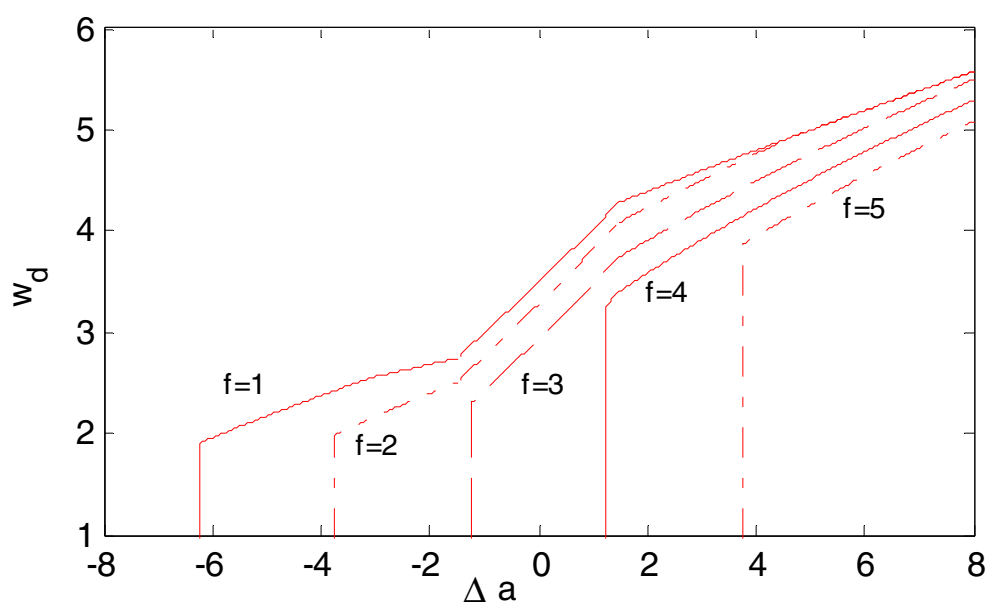

Figure 3. The wholesale price for the dominant retailer versus demand disruption with $\Delta c=0$.

the market demand increase greatly or the service cost is small, the manufacturer will decrease rapidly the subsidy rate to obtain as much profit from the dominant retailer as possible. In fact, we can find that the curve of subsidy rate in case 1 or case 3 is the very steep. However, when the demand change is very small, the subsidy rate is slightness of the disrupted amount, but only increases rapidly with the service cost.

The following Figure 3 presents the relationship between the wholesale price for the dominant retailer and the changed demand amount when the production cost is unchanged.

Figure 3 indicates that in order to induce the retail pricing of the dominant retailer to be equal with that of the centralized channel, the wholesale price has the following characteristics. First, the optimal wholesale price is a piecewise continuous and decreasing function of changed demand amount $(\Delta a)$, that is, the larger the disrupted market scale, the higher the wholesale price for each feasible service cost will be. This is mainly because the increasing of the market demand yields more profit for the dominant retailer; and then the higher wholesale price provided by the manufacturer can also secure the dominant retailer's cooperation according to the coordination mechanism. Second, the optimal wholesale price is decreasing with the service cost, that is, the higher the service cost $(f)$, the less the wholesale price for each feasible service cost will be. It implies that the manufacturer should provide more favorable wholesale price to the dominant retailer to ensure that the dominant retailer can provide the corresponding demand-stimulating service according to the coordination mechanism.

Figure 4 presents the relationship between the wholesale price for each fringe retailer and the changed demand amount when the production cost is unchanged. 


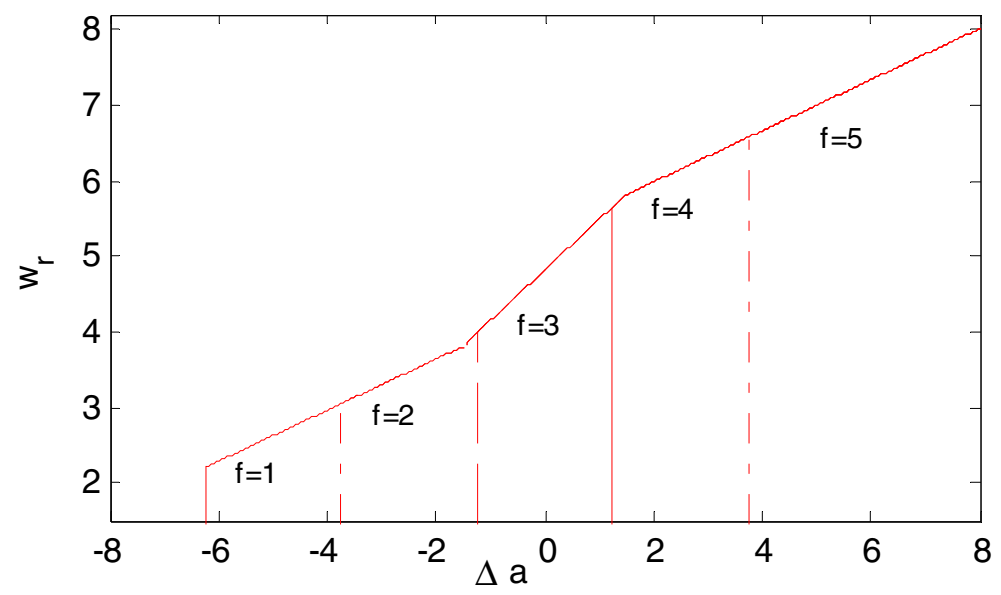

Figure 4. The wholesale price for each fringe retailer versus demand disruption with $\Delta c=0$.

Comparing Figures 3 and 4, we can find that the wholesale price for each fringe retailer has the similar managerial insights with that for the dominant retailer. Additionally, from Figure 4, the optimal wholesale price for each fringe retailer is independent of the service cost. That is, the curves of the wholesale price for each fringe retailer remain same except that the feasible regions of the disruption amount become smaller when the demand-stimulating service cost increases.

In the following, we will investigate how effects of disruption cost and demandstimulating service cost on the channel's decisions and the coordinated Grove wholesale price scheme.

Figure 5 investigate the effects different disruption amount of production cost on the retailers' optimal share of channel's profit when the disrupted amount of demand is $\Delta a=3$. In general, the share of channel's profit is decreasing with the changed amount of production cost except for the robust scale. In the robust scale, the share of channel's profit is independent of the disrupted cost. In Figure 6, we illustrates how the cost disruption, demand-stimulating service cost affect the optimal subsidy rate when the disrupted amount of demand is $\Delta a=3$.

From Figure 6, we find that with the increase of the production cost or the service cost, the manufacturer will improve the subsidy rate the dominant retailer instead. Why does the counter-intuitive case appear? In fact, the increase of production cost or service cost directly results in low profit for the channel, the manufacturer should increase subsidy rate to ensure that the dominant retailer has interest to cooperation and provision of retail services.

Figure 7 investigates how the changed amount of unit production cost and service cost affect the wholesale price for the dominant retailer when the changed demand amount is $\Delta a=3$. From Figure 7 , we find that the wholesale price increases 


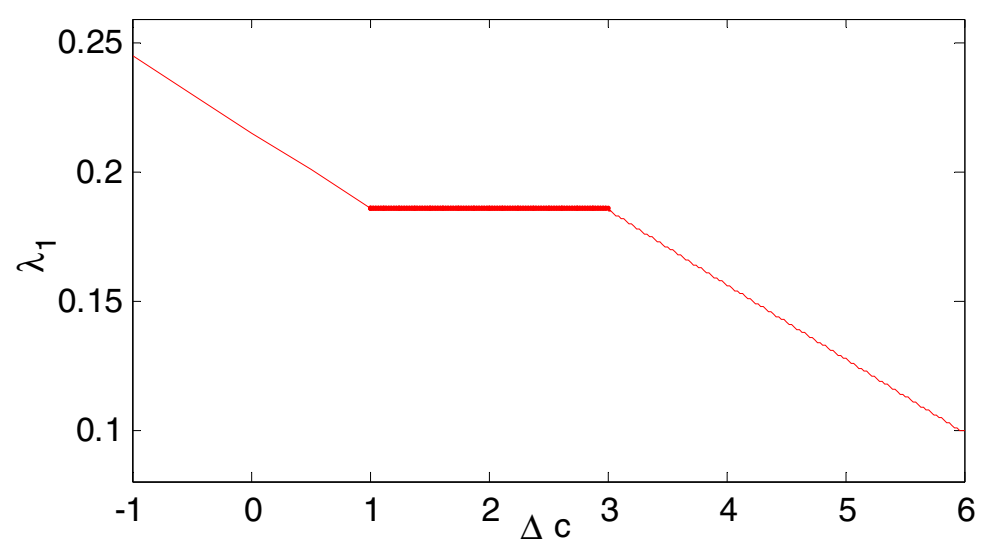

Figure 5. Optimal share of the dominant retailer versus production cost disruption with $\Delta a=3$.

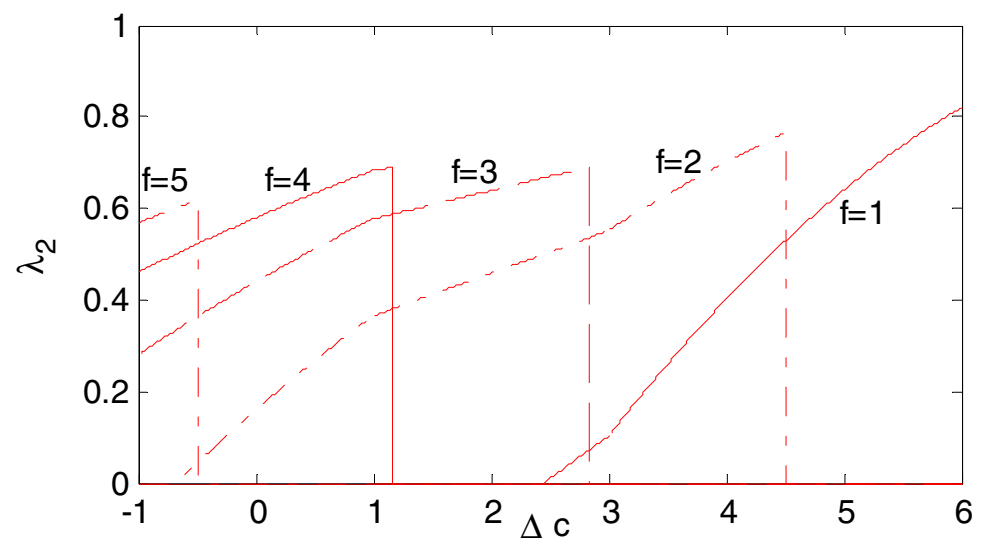

Figure 6. Optimal share of the dominant retailer versus production cost disruption with $\Delta a=3$.

with the disrupted unit cost. That is, with the increase of unit production cost, the manufacturer will raise price to the dominant retailer naturally. However, in order to ensure that the dominant retailer provision of retail services, the manufacturer will appropriately cut down the price when the service cost increase. Additionally, the wholesale price for each fringe retailer is independent of the disrupted production cost, and we do not provide the numerical analysis here.

\section{Conclusions}

In our study, a mathematic model based game theory has been proposed for analyzing and designing of coordination mechanism of a supply chain with one 


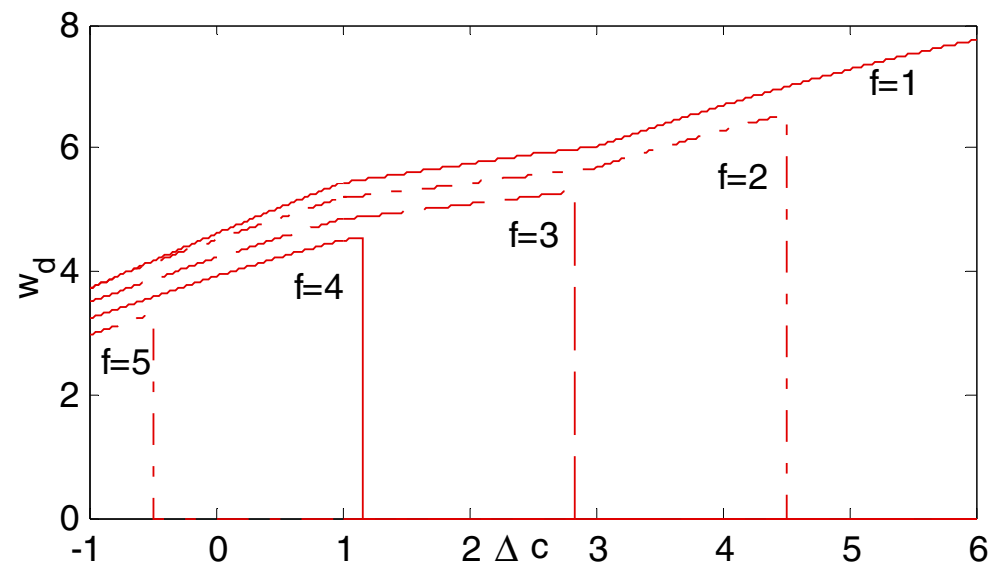

FiguRE 7 . The wholesale price for the dominant retailer versus production cost disruption with $\Delta a=3$.

dominant retailer. Differing from current literatures on the term of 'dominant', the dominant retailer in our paper does not have priority for action, but has market power in the retail market. We also assume that only the dominant retailer can provide the demand-stimulating service. This paper investigates how to design the mechanism to coordinate such a supply chain. Furthermore, we also consider the form of coordination mechanism for such a supply chain with disruptions from both demand side and production cost side, and investigate how the demandstimulating service affects the decision of dominant retailer and how to design the coordination mechanism by addition of the demand-stimulating service even if both demand and production cost are disrupted.

We show that with the nonlinear Grove wholesale price scheme, the centralized optimal supply-chain profit could be achieved under the decentralized decision. We find that the coordinated nonlinear Grove wholesale price scheme has the following characteristics. The coordination mechanism induces the retail pricing of dominant retailer to be equal with that of the channel, simultaneously, ensures that fringe retailers are not priced out of the market. The coordination mechanism ensures that the manufacturer uses minimum incentives to induce the dominant retailer to sell its product as well as providing the demand-stimulating service. Additionally, the optimal wholesale price for the dominant retailer is decreasing with the disrupted demand, but increasing with the disrupted production cost, while the wholesale price for each fringe retailer is independent of disrupted production cost. Additionally, the share of dominant retailer and the subsidy rate provided by the manufacturer are unity of opposites.

There are several directions that this research could continue. For examples, (i) we can relax some assumptions such that there are several dominant retailers who compete in price or service, and investigate how to coordinate such a supply 
chain by the nonlinear Grove wholesale price. (ii) Since we assume that the demand scale is exogenously given, the second direct extension of the paper will be the case for the uncertain demand scale. (iii) We assume that the disruption information is complete between the manufacturer and the dominant retailer, and one can also study our game model with asymmetric disruption information and study how to design an incentive mechanism revealing private information.

Acknowledgements. The authors thank the anonymous referees and an editor for their numerous constructive comments and encouragement in developing the paper. This work was supported by the Fundamental Research Funds for the Central Universities (NZ2012311).

\section{Appendix A}

Proof of Theorem 4.1.

Part (i). Since the dominant retailer's objective function is aligned with the channel's, his profit is $\pi_{d}=\lambda_{1}\left(a-b p+s_{f}\right)(p-c)-\left(1-\lambda_{2}\right) f$, and then it follows that the optimal retail price is $p^{*}$, and the channel demand is $q_{T}^{*}$. Thus, the supply chain with one dominant retailer can be coordinated, and the profit of the dominant retailer is obviously increasing with $\lambda_{1}$ linearly.

Part (ii). The profit of each fringe retailer can be denoted as follows:

$\pi_{r}=\left[p^{*}-w\left(q_{r}^{*}, s_{f}\right)\right] q_{r}^{*}=\left\{p^{*}-\left(1-\frac{\lambda_{1}}{\gamma}\right)\left[\frac{a+s_{f}}{b}-\frac{(1-\gamma)}{N b \gamma}\left(a+s_{f}-b p^{*}\right)\right]\right\} q_{r}^{*}$,

which is increasing with $\lambda_{1}$ linearly since $p^{*}>c$ is require to ensure there is non-negative unit profit in the channel.

Part (iii). In order to ensure all the retailers obtain nonnegative profits, the manufacturer will choose a proper $\lambda$ to achieve it. Obviously, either the dominant retailer or each fringe retailer obtains the reservation utility. If the dominant retailer gains zero profit, each fringe retailer will obtain a negative profit. Thus, we let

$$
w\left(q_{r}, \gamma \mid \lambda_{1}, \lambda_{2}\right)=\left(1-\frac{\lambda_{1}}{\gamma}\right)\left(\frac{a+s_{f}}{b}-\frac{q_{r}}{b \gamma}\right)=p^{*}
$$

and then we can obtain $\lambda_{1}^{*}=\gamma \frac{(N \gamma+\gamma-1)\left(a-b c+s_{f}\right)}{(2 N \gamma+\gamma-1)\left(a+s_{f}\right)+b c(1-\gamma)}$. The difference between the dominant retailer's maximal profit when the service is provided and that when it is not is given by

$$
\pi_{d}^{*}-\pi_{d, n}^{*}=\lambda_{1}^{*} \frac{s_{f}\left[s_{f}+2(a-b c)\right]}{4 b}-\left(1-\lambda_{2}\right) f .
$$

When $0<f \leqslant \lambda_{1}^{*} \frac{s_{f}\left[s_{f}+2(a-b c)\right]}{4 b}$, the manufacturer may not provide the subsidy to the retailer since the service cost is less than the revenue obtained by the 
dominant retailer. And when $f>\lambda_{1}^{*} \frac{s_{f}\left[s_{f}+2(a-b c)\right]}{4 b}$, in order to ensure that the profit of the dominant retailer is nonnegative, the manufacturer should provide a certain subside to the retailer. Therefore, we can obtain the optimal subsidy rate provided by the manufacturer to the dominant retailer, which can be denoted as following:

$$
\lambda_{2}^{*}= \begin{cases}0 & 0 \leqslant f \leqslant \lambda_{1}^{*} \frac{s_{f}\left[s_{f}+2(a-b c)\right]}{4 b} \\ 1-\lambda_{1}^{*} \frac{s_{f}\left[s_{f}+2(a-b c)\right]}{4 b f} & \lambda_{1}^{*} \frac{s_{f}\left[s_{f}+2(a-b c)\right]}{4 b} \leqslant f \leqslant f_{0} .\end{cases}
$$

Part (iv). Performing some algebraic manipulation, we can find both $\lambda_{1}^{*}$ and $\lambda_{2}^{*}$ is increasing with $\gamma$, obviously.

Proof of Theorem 5.4. Since we assume that the fringe retailers will not receive a lump sum compensation cost and simply pay a variable fee per unit $\tilde{w}\left(\tilde{q}, s_{f} \mid \tilde{\lambda}\right)$ without the second part because they do not provide service. Additionally, the manufacturer's wholesales price schedule must ensure that the fringe retailers are not priced out of the market and that the manufacturer uses minimum incentives to induce the dominant retailer to service its product. Therefore, the wholesale price for each symmetrical fringe retailer should satisfy the following

$$
w\left(\tilde{q}_{r, \text { case } 1}^{*}\right)=\left(1-\frac{\tilde{\lambda}}{\gamma}\right)\left[\frac{\tilde{a}+s_{f}}{b}-\frac{(1-\gamma)}{N b \gamma}\left(\tilde{a}+s_{f}-b \tilde{p}_{\text {case } 1}^{*}\right)\right]=\tilde{p}_{\text {case } 1}^{*}
$$

Solving the above equations, we can obtain that

$$
\tilde{\lambda}_{1, \text { case } 1}^{*}=\gamma \frac{(N \gamma+\gamma-1)\left(\tilde{a}+s_{f}-b c_{u}-b \tilde{c}\right)}{(2 N \gamma+\gamma-1)\left(\tilde{a}+s_{f}\right)+(1-\gamma)\left(b c_{u}+b \tilde{c}\right)} .
$$

When the service is provided, we let the profit of the dominant retailer's maximal profit be

$$
\tilde{\pi}_{d}=\tilde{\lambda}_{1, \text { case } 1}^{*}\left[(\tilde{a}-b \tilde{p}+s)(\tilde{p}-\tilde{c})-c_{u}\left(\tilde{a}-b \tilde{p}+s_{f}-q_{T}^{*}\right)^{+}\right]-\left(1-\tilde{\lambda}_{2}\right) f,
$$

with

$$
\tilde{p}=\frac{a+\Delta a+s_{f}+b c+b \Delta c+b c_{u}}{2 b} .
$$

When the service is not provided, we let the profit of dominant retailer's maximal profit be

$$
\tilde{\pi}_{d, n}=\tilde{\lambda}_{1, \text { case } 1}^{*}\left[(\tilde{a}-b \tilde{p})(\tilde{p}-\tilde{c})-c_{u}\left(\tilde{a}-b \tilde{p}-q_{T, n}^{*}\right)^{+}\right]
$$

with

$$
\tilde{p}=\frac{a+\Delta a+b c+b \Delta c+b c_{u}}{2 b} .
$$


Performing some algebraic manipulation, we have the difference between the dominant retailer's maximal profits when the service is provided and that when it is not, which can be denoted as following.

$$
\pi_{d}^{*}-\pi_{d, n}^{*}=\tilde{\lambda}_{1, \text { case } 1}^{*} \frac{s_{f}\left[s_{f}+2(\tilde{a}-b \tilde{c})\right]}{4 b}-\left(1-\lambda_{2}\right) f .
$$

When $0<f \leqslant \tilde{\lambda}_{1, \text { case } 1}^{*} \frac{s_{f}\left[s_{f}+2(\tilde{a}-b \tilde{c})\right]}{4 b}$, the manufacturer may not provide the subsidy to the retailer since the service cost is less than the revenue obtained by the dominant retailer. And when $f>\tilde{\lambda}_{1, \text { case } 1}^{*} \frac{s_{f}\left[s_{f}+2(\tilde{a}-b \tilde{c})\right]}{4 b}$, in order to ensure that the profit of the dominant retailer is nonnegative, the manufacturer should provide a certain subside to the retailer. Therefore, we can obtain the optimal subsidy rate provided by the manufacturer to the dominant retailer, which can be denoted as following:

$$
\tilde{\lambda}_{2, \text { case } 1}^{*}= \begin{cases}0 & 0 \leqslant f \leqslant \tilde{\lambda}_{1, \operatorname{case} 1}^{*} \frac{s_{f}\left[s_{f}+2(\tilde{a}-b \tilde{c})\right]}{4 b} \\ 1-\tilde{\lambda}_{1, \operatorname{case} 1}^{*} \frac{s_{f}\left[s_{f}+2(\tilde{a}-b \tilde{c})\right]}{4 b f} & \tilde{\lambda}_{1, \operatorname{case} 1}^{*} \frac{s_{f}\left[s_{f}+2(\tilde{a}-b \tilde{c})\right]}{4 b}<f \leqslant \tilde{f}_{0} .\end{cases}
$$

Proof of Proposition 5.5. The proof is obvious, here omitted.

Proof of Proposition 5.6. For case 2, the wholesale price for each symmetrical fringe retailer should satisfy the following

$$
w\left(\tilde{q}_{r, \text { case } 2}^{*}\right)=\left(1-\frac{\tilde{\lambda}}{\gamma}\right)\left[\frac{\tilde{a}+s_{f}}{b}-\frac{(1-\gamma)}{N b \gamma}\left(\tilde{a}+s_{f}-b \tilde{p}_{\text {case } 2}^{*}\right)\right]=\tilde{p}_{\text {case } 2}^{*} .
$$

Therefore, we can obtain the optimal share of the dominant retailer as following

$$
\tilde{\lambda}_{1, \text { case } 2}^{*}=\gamma \frac{(N \gamma+\gamma-1)\left(a+s_{f}-b c\right)}{(2 N \gamma+\gamma-1)\left(a+s_{f}\right)+2 \Delta a N \gamma+(1-\gamma) b c} .
$$

When the service is provided, we let the profit of the dominant retailer's maximal profit be

$$
\tilde{\pi}_{d}=\tilde{\lambda}_{1, \text { case } 2}^{*}\left[\left(\tilde{a}-b \tilde{p}+s_{f}\right)(\tilde{p}-\tilde{c})\right]-\left(1-\tilde{\lambda}_{2}\right) f
$$

with

$$
\tilde{p}=\left(\tilde{a}+\Delta a+s_{f}+b c\right) /(2 b) .
$$

When the service is not provided, we let the profit of the dominant retailer's maximal profit be $\tilde{\pi}_{d, n}=\tilde{\lambda}_{1, \text { case } 2}^{*}[(\tilde{a}-b \tilde{p})(\tilde{p}-\tilde{c})]$ with $\tilde{p}=(\tilde{a}+\Delta a+b c) /(2 b)$.

The difference between the dominant retailer's maximal profit when the service is provided and that when it is not is given by

$$
\pi_{d}^{*}-\pi_{d, n}^{*}=\tilde{\lambda}_{1, \mathrm{case} 2}^{*} \frac{s_{f}\left[s_{f}+2(\tilde{a}-b \tilde{c})\right]}{4 b}-\left(1-\lambda_{2}\right) f .
$$


Therefore, we have the following subsidy rate.

$$
\tilde{\lambda}_{2, \text { case } 2}^{*}= \begin{cases}0 & 0 \leqslant f \leqslant \tilde{\lambda}_{1, \text { case } 2}^{*} \frac{s_{f}\left[s_{f}+2(\tilde{a}-b \tilde{c})\right]}{4 b} \\ 1-\tilde{\lambda}_{1, \text { case } 2}^{*} \frac{s_{f}\left[s_{f}+2(\tilde{a}-b \tilde{c})\right]}{4 b f} & \tilde{\lambda}_{1, \text { case } 2}^{*} \frac{s_{f}\left[s_{f}+2(\tilde{a}-b \tilde{c})\right]}{4 b}<f \leqslant \tilde{f}_{0} .\end{cases}
$$

Proof of Theorem 5.8. Under case 3, the wholesale price for each symmetrical fringe retailer should satisfy the following

$$
w\left(\tilde{q}_{r, \text { case } 3}^{*}\right)=\left(1-\frac{\tilde{\lambda}}{\gamma}\right)\left[\frac{\tilde{a}+s_{f}}{b}-\frac{(1-\gamma)}{N b \gamma}\left(\tilde{a}+s_{f}-b \tilde{p}_{\text {case } 3}^{*}\right)\right]=\tilde{p}_{\text {case } 3}^{*} .
$$

Solving the above equations, we can obtain that

$$
\tilde{\lambda}_{1, \text { case } 3}^{*}=\gamma \frac{(N \gamma+\gamma-1)\left(\tilde{a}+s-b \tilde{c}+b c_{s}\right)}{(2 N \gamma+\gamma-1)(\tilde{a}+s)+(1-\gamma)\left(b \tilde{c}-b c_{s}\right)} .
$$

The difference between the dominant retailer's maximal profit when the service is provided and that when it is not is given as $\pi_{d}^{*}-\pi_{d, n}^{*}=\tilde{\lambda}_{1, \text { case } 3}^{*} \frac{s[s+2(\tilde{a}-b \tilde{c})]}{4 b}-(1-$ $\left.\lambda_{2}\right) f$.

Therefore, we can obtain the optimal subsidy rate provided by the manufacturer to the dominant retailer, which can be denoted as following:

$$
\tilde{\lambda}_{2, \text { case } 3}^{*}= \begin{cases}0 & 0 \leqslant f \leqslant \tilde{\lambda}_{1, \operatorname{case} 3 \frac{s[s+2(\tilde{a}-b \tilde{c})]}{4 b}}^{*} \\ 1-\tilde{\lambda}_{1, \operatorname{case} 3}^{*} \frac{s[s+2(\tilde{a}-b \tilde{c})]}{4 b f} \tilde{\lambda}_{1, \text { case } 3}^{*} \frac{s[s+2(\tilde{a}-b \tilde{c})]}{4 b}<f \leqslant \tilde{f}_{0} .\end{cases}
$$

Proofs of Proposition 5.9 is obvious, here omitted.

Proof of Proposition 5.10. Here, we only give the proof the inequality denoted as $\tilde{\lambda}_{1, \text { case } 2}^{*}<\gamma$.

$\tilde{\lambda}_{1, \text { case } 2}^{*}<\gamma$ is equal with the inequality denoted as $(N \gamma+\gamma-1)\left(a+s_{f}-b c\right)<$ $(2 N \gamma+\gamma-1)\left(a+s_{f}\right)+2 \Delta a N \gamma+(1-\gamma) b c$. It can be changed into $a+s_{f}+b c+2 \Delta a>$ 0 , which can be satisfied by $\tilde{a}>0$ and Assumption 5.1 .

\section{REFERENCES}

[1] D. Aussadavut, F. Ming, J. Apurva and M. Kamran, A supply chain model with direct and retail channels. Eur. J. Oper. Res. 187 (2008) 691-718.

[2] M. Bergen and G. John, Understanding cooperative advertising participation rates in conventional channels. J. Mark. Res. 35 (1997) 357-369.

[3] F. Bernstein and A. Federgruen, Pricing and replenishment strategies in a distribution system with competing retailers. Oper. Res. 51 (2003) 409-426.

[4] T. Boyaci and G. Gallego, Coordinating pricing and inventory replenishment policies for one wholesaler and one or more geographically dispersed retailers. Int. J. Prod. Econ. 77 (2002) 95-111. 
[5] G.P. Cachon, Supply chain coordination with contracts, in Handbook in operation research and management science: supply chain management, edited by S. Graves and T. de Kok. North-Holland (2003).

[6] G.P. Cachon and M.A. Lariviere, Supply chain coordination with revenue sharing contracts: Strengths and limitations. Manage. Sci. 51 (2005) 30-44.

[7] J. Causen, J. Hansen and J. Larsen, Disruption management. OR/MS Today 28 (2001) $40-43$.

[8] M. Celikbas, J.G. Shanthikumar and J.M. Swaminathan, Coordinating production quantities and demand forecasts through penalty schemes. IIE Trans. 31 (1999) 851-864.

[9] F. Chen, Coordination mechanisms for a distribution system with one supplier and multiple retailers. Manage. Sci. 47 (2001) 698-708.

[10] T.H. Chen, Coordinating the ordering and advertising policies for a single-period commodity in a two-level supply chain. Comput. Ind. Eng. 61 (2011) 1268-1274.

[11] J. Chen and P.C. Bell, Coordinating a decentralized supply chain with customer returns and price-dependent stochastic demand using a buyback policy. Eur. J. Oper. Res. 212 (2011) 293-300.

[12] K.B. Chen and T.J. Xiao, Demand disruption and coordination of the supply chain with a dominant retailer. Eur. J. Oper. Res. 197 (2009) 225-234.

[13] K.B. Chen and T.J. Xiao, Ordering policy and coordination of a supply chain with twoperiod demand uncertainty. Eur. J. Oper. Res. 215 (2011) 347-357.

[14] P.K. Chintagunta, Investigating the sensitivity of equilibrium profits to advertising dynamics and competitive effects. Manage. Sci. 39 (1993) 1146-1162.

[15] J. Clausen, J. Hansen and J. Larsen, Disruption management. OR/MS Today 28 (2001) $40-43$.

[16] A.J. Dukes, E. Gal-Or and K. Srinivasan, Channel bargaining with retailer asymmetry. J. Mark. Res. 43 (2006) 84-97.

[17] L.T. Friesz, I. Lee and C.-C. Lin, Competition and disruption in a dynamic urban supply chain. Transp. Res. Part B 45 (2011) 1212-1231.

[18] T. Groves and M. Loeb, Incentives in a divisionalized firm. Manage. Sci. 25 (1979) 221-230.

[19] H. Gurnani and Y. Gerchak, Coordination in decentralized assembly systems with uncertain component yields. Eur. J. Oper. Res. 176 (2007) 1559-1576.

[20] X. He, A. Prasad and S. Sethi, Cooperative advertising and pricing in a dynamic stochastic supply chain: Feedback Stackelberg strategies. Prod. Oper. Manag. 18 (2009) 78-94.

[21] Z.M. Huang, S.X. Li and V. Mahajan, An analysis of manufacturer-retailer supply chain coordination in cooperative advertising. Decis. Sci. 33 (2002) 469-96.

[22] S. Huang, C. Yang and X. Zhang, Pricing and production decisions in dual-channel supply chains with demand disruptions. Comput. Ind. Eng. 62 (2012) 70-83.

[23] S. Jorgensen, S.P. Sigue and G. Zaccour, Dynamic cooperative advertising in a channel. J. Retail. 76 (2000) 71-92.

[24] S. Karray and G. Zaccour, Could co op advertising be a manufacturer's counterstrategy to store brands? J. Bus. Res. 59 (2006) 1008-1015.

[25] S. Karray and G. Zaccour, Effectiveness of coop advertising programs in competitive distribution channels. International Game Theory Review 9 (2007) 151-167.

[26] P.R. Kleindorfer, J.C. Belke, M.R. Elliot, K. Lee, R.A. Lowe and H. Feldman, accident epidemiology and the U.S. chemical industry: accident history and worst-case data from RMP*Info. Risk Anal. 23 (2003) 865-881.

[27] M.A. Lariviere, Supply chain contracting and coordination with stochastic demand, in Quantitative models for supply chain management, edited by S. Tayur, R. Ganeshan and M. Magazine. Kluwer Academic Publishers, Boston, MA (1999) 233-268.

[28] A.H.L. Lau, H.S. Lau and J.C. Wang, How a dominant retailer might design a purchase contract for a newsvendor-type product with price-sensitive demand. Eur. J. Oper. Res. 190 (2008) 443-458.

[29] Y. Li, X.J. Xu and F. Ye, Supply chain coordination model with controllable lead time and service level constraint. Comput. Ind. Eng. 61 (2011) 858-864. 
[30] R.Y. Lin and L.Z. Kong, The impact of channel power of symmetric competing channels on the profit segmentation, in Processing of National Conference of Chinese National Academy (2002).

[31] M. Maier, How to Beat Wal-Mart, in Business, 2.0 (2005).

[32] X.T. Qi, J. Bard and G. Yu, Supply chain coordination with demand disruptions. Omega 32 (2004) 301-312.

[33] J. Raju and S.J. Zhang, Channel coordination in the presence of a dominant retailer. Mark. Sci. 24 (2005) 254-262.

[34] M.H. Riordan, Anticompetitive vertical integration by a dominant firm. Amer. Econom. Rev. 88 (1998) 1232-1248.

[35] L.S, Robert, GM contains its quarterly loss at $\$ 809$ million. Wall Street J. A2 (1998).

[36] M. Seyed Esfahani, M. Biazaran and M. Gharakhani, A game theoretic approach to coordinate pricing and vertical co op advertising in manufacturer-retailer supply chains. Eur. J. Oper. Res. 211 (2011) 263-273.

[37] W. Shepherd, The economics of industrial organization. Prentice Hall, Upper Saddle River, NJ (1997) 62-64.

[38] L.V. Snyder, P.M. Scaparra, M.S. Daskin and R.L. Church, Planning for disruptions in supply chain networks, in Tutorials in operations research. INFORMS (2006) 234-257.

[39] J.G. Szmerekovsky and J. Zhang, Pricing and two-tier advertising with one manufacturer and one retailer. Eur. J. Oper. Res. 192 (2009) 904-917.

[40] A. Tsay, Risk sensitivity in distribution channel partnerships: Implications for manufacturer return policies. J. Retail. $\mathbf{7 8}$ (2002) 147-160.

[41] A. Tsay and N. Agrawal, Channel dynamics under price and service competition. Manuf. Serv. Oper. Manag. 2 (2000) 372-391.

[42] A. Tsay, S. Nahmias and N. Agrawal, Modeling supply chain contracts: a review, in edited by S. Tayur, M. Magazine and R. Ganeshan. Kluwer Academic Publishers Quantitative Models for Supply Chain Management, MA (1998) 197-232.

[43] J. Useem, How retelling's superpower-and our biggest most admired company- is changing the rules for corporate America. Fortune (2003) 65.

[44] S. Weinstein, The price is righter. Progressive Grocer 79 (2000) 89-94.

[45] Y.S. Xia, M. Yang, B. Golany, S. Gilbert and G. Yu, Real-time disruption management in a two-stage production and inventory system. IIE Trans. 36 (2004) 111-125.

[46] T.J. Xiao, G. Yu, Z.H. Sheng and Y.S. Xia, Coordination of a supply chain with onemanufacturer and two-retailers under demand promotion and disruption management decisions. Ann. Oper. Res. 135 (2005) 87-109.

[47] J.X. Xie and A. Neyret Co op advertising and pricing models in manufacturer-retailer supply chains. Comput. Ind. Eng. 56 (2009) 1375-1385.

[48] J.X. Xie and J.C. Wei, Coordinating advertising and pricing in a manufacturer-retailer channel. Eur. J. Oper. Res. 197 (2009) 785-791.

[49] H. Yu, C. Song and J. Chen, Simulating the supply chain disruption for the coordination supply chain. J. Syst. Sci. Syst. Eng. 16 (2007) 323-335.

[50] X. Zou, S. Pokharel and R. Piplani, Channel coordination in an assembly system facing uncertain demand with synchronized processing time and delivery quantity. Int. J. Prod. Econ. 42 (2004) 4673-4689. 